\title{
Only limited evidence available for the effectiveness and cost effectiveness of dental auxiliaries
}

\section{Abstracted from}

\section{Wright JT, Graham F, Hayes C, et al.}

A systematic review of oral health outcomes produced by dental teams incorporating midlevel providers. J Am Dent Assoc 2013; 144: 75-91.

Address for correspondence: Dr. Frantsve-Hawley, Research Institute and Center for Evidence-Based Dentistry, Division of Science, American Dental Association, $211 \mathrm{E}$. Chicago Ave., Chicago, III. 60611, USA. E-mail: frantsvej@ada.org

\section{Question: In populations where non-dentists conduct diagnostic, treatment planning, and/ or irreversible/surgical dental procedures, is there a change in disease increment, untreated dental disease, and/or cost-effectiveness in dental care?}

Data sources Medline, Embase, CINAHL, LILACS, Cochrane Database of Systematic Reviews, OpenGrey (System for Information on Grey Literature in Europe [SIGLE]-based), Scirus, Science.gov, Cost-Effective Analysis (CEA) Registry, European Network of Health Economics Evaluation Databases (EURON-HEED), ClinicalTrials.gov and Health Services Research Projects in Progress (HSRProj) databases. They also contacted 20 separate organisations.

Study selection All study designs were considered with no limits on dates, age of study, language or country. Government reports, peer-reviewed publications, dissertations and theses were included. Editorials, opinion pieces, educational pieces, narrative reviews, abstracts without full-text availability and raw data such as those from national oral health surveys were excluded. Study quality and risk of bias was assessed.

Data extraction and synthesis Data extraction was conducted independently, and meta-analysis was planned for the data, but only a qualitative synthesis could be conducted.

Results Eighteen observational studies were included, 13 were considered to be at high risk of bias, five at moderate risk and one at low risk. They were conducted in Australia, Canada, Hong Kong, New Zealand and the United States. All the studies were related to dental caries with only studies involving dental nurses and therapists meeting the inclusion criteria. No studies regarding cost effectiveness, irreversible diagnostic procedures or diseases other than caries were in included.

Conclusions The authors concluded that the quality of the evidence was poor. They found that in select groups in which participants received irreversible dental treatment from teams that included midlevel providers, caries increment, caries severity or both decreased across time; however, there was no difference in caries increment, caries severity or both compared with those in populations in which dentists provided all irreversible treatment. In select groups in which participants had received irreversible dental treatment from teams that included midlevel providers, there was a decrease in untreated caries across time and a decrease in untreated caries compared with that in populations in which dentists provided all treatment.

\section{Commentary}

Access to dental care is difficult in many parts of the world, not least in developing and underdeveloped countries, but it can also be a problem in developed counties in some urban and rural communities. Training dentists is a long and expensive endeavour so alternative providers have been developed in some countries. These providers perform some of the reversible and irreversible procedures traditional performed by dentists. Both the names (dental assistant, dental auxiliary, dental nurse, extended duties dental nurse, dental hygienist, dental technician, dental therapist) and the range of duties they perform vary widely from country to country. Collectively they were referred to as professions complementary to dentistry (PCDs) in an earlier review, and in this review as midlevel providers.

The review process has been robust with searches conducted in a wide range of databases and with independent selection, data abstraction and assessment of the study quality and risk of bias being undertaken. The question chosen for this review is very interesting as it was deliberately focused on health outcomes, with two key elements being a change in the disease increment and levels of untreated dental disease. The authors touch on this in their discussion as it could be argued whether dentists themselves have much impact on disease increment, although you would anticipate they would have an impact on untreated disease. Their conclusion that were no differences between populations where dentists provided all care and those that used midlevel providers could be interpreted as; there was no difference so we should continue to use dentists, or; as they don't take as long to train we should use midlevel providers in place of dentists.

The review of PCDs conducted by Galloway et al. in 2002 addressed the question whether midlevel providers could diagnose, have the same level of technical competence as dentists for certain procedures, could perform oral health promotion, were acceptable to patients and had any impact on productivity. As with this current review the available evidence was not of a high quality but it did suggest that PCDs could perform activities they were trained for to the same level if not better than dentists.

The current review did not find any evidence for the cost-effectiveness of using midlevel providers and while the Galloway review did conduct a meta-analysis of eight studies indicating an increase in output from an extended-duty dental nurse (WMD 46\%, 95\% CI: $35,56)$, and 36\% WMD (95\% CI: 14, 59) from a dental hygienist. However the figures need to be interpreted very cautiously as the included studies were very heterogeneous. In addition, while this 


\section{EFFECTIVE PRACTICE AND ORGANISATION OF CARE}

suggests an impact on productivity, it does not indicate an impact on cost-effectiveness.

Labour substitution in health care has been happening for many years, with a range of duties previously carried out by high-level providers being transferred to lower level providers. A summary of labour substitution in health care by Sibbald et al. confirms that the main drivers for this are seen as; to compensate for shortages of (or reduce demand for) highly qualified staff, to reduce cost and improve efficiency. Their summary suggests that labour substitution is a plausible strategy for addressing workforce shortages and can reduce wage costs (under certain conditions), and can, in the right circumstances improve efficiency. They also noted a number of potential unintended consequences for patients, as where substitution increases team sizes this can have negative impacts on continuity of patient care and patient satisfaction. In addition there are implications relating to training, regulation, management of change and co-ordination of care. In dental care systems where midlevel providers are currently operating, the first two of these elements are largely in place so it is likely that management of change and coordination of care may potentially cause some challenges.

Sibbald et al. have largely drawn from work within the medical sector; the evidence from this review and the early Galloway review would suggest that we have limited evidence on which to base firm decisions about the nature and delivery of new-workforce models in dentistry. This review has suggested some useful areas for future research which may provide some answers at some point in the future, and a Cochrane review is currently underway looking at the effectiveness of dental auxiliaries in providing care traditionally provided by dentists. Although this might not turn up any additional information it may add more weight to those who have been calling for more and better research into this important area of dental service delivery.

\section{Derek Richards}

Centre for Evidence-based Dentistry Oxford, UK.

1. Galloway J, Gorham J, Lambert M, et al. The professionals complementary to dentistry: systematic review and synthesis. University College London, Eastman Dental Hospital, Dental Team Studies Unit, 2002.

2. Sibbald B, McBride A, Birch S. Labour substitution and efficiency in health care delivery: general principles and key messages. Centre for Workforce intelligence. London 2011. http://www.cfwi.org.uk/publications/labour-substitution-andefficiency-in-healthcare-delivery-general-principles-and-key-messages [Accessed March 2013].

3. Dyer T, Brocklehurst P, Glenny AM, et al. Dental auxiliaries for dental care (Protocol). Cochrane Database Syst Rev 2012; Issue 9. Art. No. CD010076. DOI: 10.1002/14651858.CD010076.

Evidence-Based Dentistry (2013) 14, 38-39. doi:10.1038/sj.ebd.6400927 